\title{
Sequência de tratamentos para lixiviado de aterro sanitário utilizando os processos físico-químico convencional e Fenton como pós-tratamento
}

\section{Landfill leachate treatment train using conventional physical chemical and Fenton processes as post treatment}

Data de entrada: 09/08/2014

Data de aprovação: 03/12/2014

Camila Lopes Maler / Luiza Reis Simionato / Thais Aparecida dos Santos / Fernando Fernandes

DOI:10.4322/dae.2014.145

Resumo:

Após tratamento por stripping e tratamento biológico por lodo ativado com nitrificação-desnitrificação, o lixiviado de aterro sanitário passou por pós-tratamento pelo processo de coagulação-floculação-sedimentação e Fenton. Nos dois casos, foi utilizada a técnica de planejamento fatorial de experimentos, tendo como variáveis independentes o $\mathrm{pH}$, a concentração de Fe (II) e, no caso do Fenton, a concentração de peróxido de hidrogênio. A otimização do processo foi definida pela variável remoção de DQQO e cor verdadeira, utilizando a metodologia de superfícies de contorno aliada à técnica de planejamento experimental. Utilizado como pós-tratamento, o processo Fenton permitiu remoção de $71 \%$ da DQO e $92 \%$ da cor verdadeira. Já o processo físico-químico levou à redução de 78\% de DQQO e 96\% da cor verdadeira.

Palavras-chave: Lixiviado de aterro sanitário. Tratamento físico-químico. Fenton.

\section{Abstract:}

After air stripping and biological treatment with activated sludge with nitrification-denitrification, the sanitary landfill leachate was pos-treated by conventional coagulation-floculation-sedimentation and Fenton processes. In both cases it was used the technique of experimental design for optimization of the variables $\mathrm{pH}$, concentration of Fe (II) and hydrogen peroxide as independent variables. The process optimization was defined by variable response COD and true colour removal, combining the technique of experimental design to surface responsemethodology. The Fenton process used as post treatment allowed a COD removal of $71 \%$ and $92 \%$ of true colour removal. The physical chemical process had an efficience of $78 \%$ of COD removal and $96 \%$ of true colour removal.

Keywords: Landfill leachate. Physical chemical process. Fenton process.

Camila Lopes Maler

Bacharel em Química e mestre pelo Programa de Pós-Graduação em Engenharia de Edificações e Saneamento da Universidade Estadual de Londrina (UEL).

Luiza Reis Simionato e Thais Aparecida dos Santos

Acadêmicas do curso de Engenharia Civil da UEL.

Fernando Fernandes

Professor associado no Centro de Tecnologia e Urbanismo da UEL. Doutor pelo INPT de Toulouse, França. Coordenador da pesquisa.

Endereço para correspondência:

E-mail: camilamalerahotmail.com

Telefones para contato: (44) 3234-4326 / (44) 9928-0269 


\section{INTRODUÇÃO}

Os resíduos sólidos domiciliares, quando aterrados, são submetidos a processos de decomposição química e biológica, dando origem a efluentes líquidos e gasosos. A fração líquida, denominada lixiviado, é formada pela umidade contida nos resíduos, produto do processo de biodegradação anaeróbia, e pelo aporte das precipitações pluviométricas e outros tipos de infiltração que percolam o aterro (KJELDSEN et al., 2002). Os lixiviados têm composição variada, porém sempre apresentam elevado potencial de contaminação, cor escura, odor desagradável, elevadas concentrações de nitrogênio amoniacal e de compostos orgânicos de difícil degradação (RENOU, 2008).

Como o lixiviado é um efluente de difícil tratamento e de composição complexa, geralmente para obter resultados que atendam aos limites ambientais de lançamento, é necessária uma sequência de processos de tratamento, cada um deles atuando sobre determinado componente poluente do lixiviado. Entre as alternativas de tratamento, uma sequência promissora é aquela que combina o arraste (stripping) do nitrogênio amoniacal com o tratamento biológico aeróbio. $\mathrm{O}$ arraste, facilitado pelo elevado $\mathrm{pH}$ do lixiviado estabilizado (aproximadamente 8,0), pode eliminar até $50 \%$ do nitrogênio amoniacal (MALER, 2012), enquanto o tratamento biológico aeróbio com nitrificação-desnitrificação pode eliminar o restante do nitrogênio e parte da matéria orgânica. No entanto, mesmo após o tratamento biológico, a matéria orgânica refratária permanece no lixiviado, o que demanda um tratamento complementar.

Nesse contexto, o processo Fenton e a técnica de coagulação-floculação-sedimentação apresentamse como alternativas de tratamento que podem ser associadas aos processos biológicos, permitindo a remoção da matéria orgânica refratária e cor.

No caso do Fenton, o mecanismo de atuação envolve a geração e emprego de espécies transitó- rias, principalmente o radical hidroxila $(\mathrm{OH})$, que apresenta alto poder oxidante e permite a mineralização completa de contaminantes tóxicos (PERALTA-ZAMORA et al., 1997). Nesse caso, o radical é gerado pela combinação de sais de ferro (II) e peróxido de hidrogênio $\left(\mathrm{H}_{2} \mathrm{O}_{2}\right)$, conforme a equação sinteticamente resumida a seguir (LANGE et al., 2006; MORAVIA et al., 2011).

$\mathrm{Fe}^{2+}+\mathrm{H}_{2} \mathrm{O}_{2} \rightarrow \mathrm{Fe}^{3+}+\cdot \mathrm{OH}+\mathrm{OH}$

(Equação 1)

A coagulação-floculação-sedimentação, por sua vez, consiste numa sequência de processos. A coagulação geralmente é realizada com a adição de sais de ferro ou de alumínio, tendo como objetivo a aglomeração de partículas em suspensão ou em estado coloidal na água residuária (DI BERNARDO, 2005). Dessa forma, o processo consiste em neutralizar as forças elétricas superficiais e anular as forças repulsivas entre as partículas por meio da adição de produtos químicos específicos. Uma vez neutralizadas as forças superficiais, o efluente é submetido à leve agitação para facilitar o contato entre os flocos sem quebrá-los, caracterizando a etapa de floculação. Os flocos maiores formados podem, então, ser removidos por sedimentação ou flotação.

O objetivo deste trabalho é comparar a aplicabilidade do processo Fenton e do tratamento físicoquímico por coagulação-floculação-sedimentação precedidos por uma sequência de tratamento que envolve a remoção de nitrogênio amoniacal por arraste de ar (stripping) e tratamento biológico por lodos ativados em bateladas sequenciais com nitrificação-desnitrificação.

\section{METODOLOGIA}

\section{Lixiviado}

O lixiviado foi coletado do aterro municipal de Rolândia - PR (cerca de 50.000 habitantes), que se encontra em atividade há nove anos e apresenta características de aterro estabilizado, com elevada cor verdadeira e alta concentração de nitrogênio 
amoniacal, além da baixa relação Demanda Biológica de Oxigênio (DBO)/Demanda Química de Oxigênio (DQO), que confere características recalcitrantes ao efluente (Tabela 1).

O lixiviado coletado foi armazenado em tanque de fibra de vidro com capacidade de $15 \mathrm{~m}^{3}$.

\section{Tratamento prévio por arraste e lodos ativados} em bateladas sequenciais

O lixiviado foi submetido ao tratamento prévio por arraste (stripping), no qual o nitrogênio amoniacal foi removido por meio de volatilização. Essa etapa foi objeto de pesquisas anteriores específicas (ALVIM, 2008; HOSSAKA, 2012; FELICl, 2013) e, neste trabalho, foi realizada em tanques de capacidade volumétrica de $1,0 \mathrm{~m}^{3}$ providos de sistema de agitação com paletas verticais em aço inoxidável e polipropileno e controle de agitação com motorredutor e inversor de frequência. Nesse tratamento, não houve alteração do $\mathrm{pH}$ inicial do lixiviado e o processo teve duração de 21 dias, perdurando até que se observasse uma redução de $40 \%$ a $50 \%$ do teor de nitrogênio amoniacal inicial.
Em seguida, o lixiviado passou pelo tratamento biológico com lodos ativados em bateladas sequenciais, realizado em duas fases. A fase aeróbia, cujo objetivo é a oxidação do nitrogênio amoniacal a nitrito (via curta), foi realizada em reator provido de sistema de injeção de ar. A duração dessa etapa foi de nove dias, correspondente ao período necessário para a redução completa de nitrogênio amoniacal a nitrito. A segunda fase, anóxica, que visa à desnitrificação, foi realizada no mesmo reator e durou 21 dias, com adição de etanol como fonte de carbono para os microrganismos envolvidos. Nessa situação específica, a duração da fase foi relativamente longa, o que pode ser atribuído às baixas temperaturas $\left(19-21^{\circ} \mathrm{C}\right)$ registradas no período de realização do experimento. Em outros experimentos, com condições de temperatura ambiente mais alta, a fase anóxica durou de um a cinco dias.

As características do lixiviado bruto e após cada etapa do tratamento são apresentadas na Tabela 1.

Observa-se que o processo de stripping e a nitrificação têm como consequências a redução con-

Tabela 1 - Valores médios dos parâmetros de caracterização do lixiviado bruto e após stripping e tratamento biológico.

\begin{tabular}{|c|c|c|c|c|}
\hline Parâmetro & Unidade & Bruto & $\begin{array}{c}\text { Após } \\
\text { stripping }\end{array}$ & Após tratamento biológico \\
\hline pH & - & 9,1 & 8,4 & 9,7 \\
\hline Alcalinidade & $\mathrm{mg} \mathrm{CaCO}_{3} / \mathrm{L}$ & 4.238 & 2259 & 1401 \\
\hline Temperatura & ${ }^{\circ} \mathrm{C}$ & \multicolumn{2}{|c|}{19,4 a 21,8} & 22,0 \\
\hline Oxigênio dissolvido & $\mathrm{mg} \mathrm{O}_{2} / \mathrm{L}$ & \multicolumn{2}{|c|}{0,5 a 0,9} & 0,3 \\
\hline Cor verdadeira & $\mathrm{uH}$ & 4.180 & - & 3.386 \\
\hline Nitrogênio amoniacal & $\mathrm{mg} \mathrm{N}-\mathrm{NH}_{3} / \mathrm{L}$ & 859 & 325 & 12 \\
\hline NKT* & $\mathrm{mg} \mathrm{N}-\mathrm{NH}_{3} / \mathrm{L}$ & 997 & 438 & 85 \\
\hline Nitrito* & $\mathrm{mg} \mathrm{N}-\mathrm{NO}_{2} / \mathrm{L}$ & 0,1 & 65,6 & 0,2 \\
\hline Nitrato* & $\mathrm{mg} \mathrm{N}-\mathrm{NO}_{3} / \mathrm{L}$ & - & 9,8 & 2,0 \\
\hline DBO* & $\mathrm{mg} \mathrm{O}_{2} / \mathrm{L}$ & 55 & 45 & 1.802 \\
\hline DQQ** & $\mathrm{mg} \mathrm{O}_{2} / \mathrm{L}$ & 1.819 & 1.816 & 2.210 \\
\hline $\mathbf{S T} *$ & $\mathrm{mg} / \mathrm{L}$ & 6.556 & 7.796 & 8.174 \\
\hline STV* & $\mathrm{mg} / \mathrm{L}$ & 5.118 & 6.067 & 6.543 \\
\hline STF* & $\mathrm{mg} / \mathrm{L}$ & 1.438 & 1.729 & 1.631 \\
\hline SST* & $\mathrm{mg} / \mathrm{L}$ & 78 & 107 & 103 \\
\hline SSV* & $\mathrm{mg} / \mathrm{L}$ & 12 & 9 & 14 \\
\hline SSF* & $\mathrm{mg} / \mathrm{L}$ & 66 & 99 & 89 \\
\hline
\end{tabular}

Notas: *NKT: Nitrogênio Kjeldahl Total. DBO: Demanda Bioquímica de Oxigênio. DQQ: Demanda Química de Oxigênio. ST: Sólidos Totais. STV: Sólidos Totais Voláteis. STF: Sólidos Totais Fixos. SST: Sólidos Suspensos Totais. SSV: Sólidos Suspensos Voláteis. SSF: Sólidos Suspensos Fixos. 
siderável de alcalinidade, pouca redução de cor e elevada remoção de nitrogênio.

\section{Processo Fenton}

O tratamento do lixiviado pelo processo Fenton foi realizado em equipamento de reatores estáticos em escala de bancada (Nova Ética - 218/6 LDBE).

Para os ensaios referentes ao processo Fenton, ajustou-se inicialmente o $\mathrm{pH}$ do lixiviado com soluções de ácido sulfúrico $5 \mathrm{~N}$ e hidróxido de sódio $5 \mathrm{~N}$ até os valores predefinidos. $\mathrm{Na}$ sequência, foram adicionados os reagentes de Fenton: sulfato de ferro seguido por peróxido de hidrogênio e agitação a 100 rpm por 60 min.

Após esses procedimentos, uma parcela do lixiviado tratado foi coletada para análises sem neutralização. $O$ efluente foi deixado em repouso para sedimentação por $30 \mathrm{~min}$. Posteriormente, as amostras foram neutralizadas com hidróxido de sódio $5 \mathrm{~N}$ e analisadas para determinação dos parâmetros monitorados.

\section{Coagulação-floculação-sedimentação}

De maneira similar ao processo anterior, o tratamento do lixiviado pela técnica de coagulação-floculação-sedimentação foi realizado em equipamento de reatores estáticos em escala de bancada (Nova Ética - 218/6 LDBE).

Como coagulante foi utilizado o cloreto férrico $\left(\mathrm{FeCl}_{3}\right)$ e os ajustes de $\mathrm{pH}$ foram realizados com soluções de ácido clorídrico $(\mathrm{HCl} 20 \%)$ e hidróxido de sódio ( $\mathrm{NaOH} 5 \mathrm{~N})$.

Estudos anteriores (FELICl, 2013) mostraram que não houve influência das variáveis gradiente de velocidade de mistura rápida (Gmr) e gradiente de velocidade de floculação (Gfloc) quando se analisaram a remoção de cor e a DQQ de lixiviados com características semelhantes. Dessa forma, os ensaios foram realizados sob condições fixas dessas variáveis: gradiente médio de velocidade de mistura rápida e gradiente médio de velocidade de floculação foram mantidos em $600 \mathrm{~s}^{-1}$ e $20 \mathrm{~s}^{-1}$, respectivamente; o tempo médio de mistura rápida (Tmr) foi de $1 \mathrm{~min}$; e o tempo médio de floculação (Tfloc) foi de 20 min. As variáveis independentes estudadas foram apenas a concentração de coagulante e o $\mathrm{pH}$ do lixiviado.

\section{Análises}

Antes e após o tratamento, foram analisados os parâmetros apresentados na Tabela 2, seguindo os protocolos apresentados pela American Public Health Association (APHA) e pela American Water Works Association (AWWA) (APHA; AWWA, 2005).

O peróxido de hidrogênio residual foi determinado pelo método iodométrico, visando a eliminar sua interferência no valor de DQO. Por esse método, a DQO do peróxido de hidrogênio é determinada pela Equação 2; na sequência, esse valor é descontado da DQO total da amostra (Equação 3) (KANG et al., 1999).

Tabela 2 - Parâmetros e métodos de caracterização do lixiviado.

\begin{tabular}{|c|c|c|}
\hline \multicolumn{1}{|c|}{ Parâmetro } & Método padrão & Método \\
\hline pH & - & Método potenciométrico \\
\hline Alcalinidade & $2320 \mathrm{~B}$ & Método titulométrico \\
\hline Sólidos totais & $2540 \mathrm{~B}$ & Sólidos totais secos a $103-105{ }^{\circ} \mathrm{C}$ \\
\hline Sólidos em suspensão & $2540 \mathrm{E}$ & Sólidos voláteis a $550^{\circ} \mathrm{C}$ \\
\hline Nitrogênio amoniacal & $4500-\mathrm{NH3} \mathrm{B} \mathrm{e} \mathrm{C}$ & Destilação e titulação \\
\hline NKT & $4500-N o r g ~ B$ & Macro-Kjedhal \\
\hline Nitrito & $4500 N O 2-\mathrm{B}$ & Método colorimétrico \\
\hline Nitrato & - & Método do ácido salicílico \\
\hline DQO & $5220 \mathrm{C}$ & Método do refluxo fechado \\
\hline DBO & $5210 \mathrm{~B}$ & Teste de DBO 5 dias \\
\hline
\end{tabular}


$\mathrm{DQOH}_{2} \mathrm{O}_{2}\left(\mathrm{mg} \mathrm{L}^{-1}\right)=0,4706\left[\mathrm{H}_{2} \mathrm{O}_{2}\right]-4,06 \times 105$

$$
\left[\mathrm{H}_{2} \mathrm{O}_{2}\right]^{2}
$$

(Equação 2)

$\mathrm{DQO}_{\text {amostra }}\left(\mathrm{mg} \mathrm{L}^{-1}\right)=\mathrm{DQO}_{\text {medida }}-\mathrm{DQOH}_{2} \mathrm{O}_{2}$

(Equação 3)

\section{Planejamento fatorial de experimentos}

Para a realização e análise dos experimentos, foi aplicada a técnica de planejamento fatorial aliada à metodologia de superfície de respostas. A fim de otimizar os ensaios de bancada,, utilizaram-se como variáveis-resposta a porcentagem de remoção de corverdadeira e a porcentagem de remoção de DQQO.
Os resultados foram analisados após cada etapa do planejamento fatorial utilizando o programa Statistica 7. Para tal, foi avaliada a porcentagem de remoção de DQQO por meio da análise do modelo matemático obtido e da superfície de resposta para essa variável.

De acordo com testes preliminares e a literatura estudada, parâmetros como a velocidade de agitação e o tempo de reação apresentam influência menos significativa sobre as variáveis-resposta. Assim, as variáveis analisadas foram a concentração de peróxido de hidrogênio e de sulfato de ferro hepta-hidratado e o $\mathrm{pH}$ inicial do lixiviado. As variáveis definidas para o estudo do processo Fenton e os respectivos níveis são apresentados na Tabela 3.

Tabela 3 - Variáveis e níveis definidos para o estudo do processo Fenton como pós-tratamento de lixiviado de aterro sanitário utilizando a técnica de planejamento fatorial de experimentos.

\begin{tabular}{|c|c|c|c|c|c|c|c|}
\hline \multirow[b]{2}{*}{ Planejamento } & \multirow[b]{2}{*}{ Ensaio } & \multicolumn{3}{|c|}{ Variável } & \multicolumn{3}{|c|}{ Variável Codificada } \\
\hline & & $\begin{array}{c}{\left[\mathrm{H}_{2} \mathrm{O}_{2}\right]} \\
\left(\mathrm{mg} \mathrm{L}^{-1}\right)\end{array}$ & $\begin{array}{c}{\left[\mathrm{Fe}^{2+}\right]} \\
\left(\mathrm{mg} \mathrm{L}^{-1}\right)\end{array}$ & $\mathrm{pH}$ & $\begin{array}{c}{\left[\mathrm{H}_{2} \mathrm{O}_{2}\right]} \\
\left(\mathrm{mg} \mathrm{L}^{-1}\right)\end{array}$ & $\begin{array}{c}{\left[\mathrm{Fe}^{2+}\right]} \\
\left(\mathrm{mg} \mathrm{L}^{-1}\right)\end{array}$ & $\mathrm{pH}$ \\
\hline \multirow{10}{*}{1} & 1 & 1.000 & 100 & 3,0 & -1 & -1 & -1 \\
\hline & 2 & 2.000 & 100 & 3,0 & +1 & -1 & -1 \\
\hline & 3 & 1.000 & 500 & 3,0 & -1 & +1 & -1 \\
\hline & 4 & 2.000 & 500 & 3,0 & +1 & +1 & -1 \\
\hline & 5 & 1.000 & 100 & 5,0 & -1 & -1 & +1 \\
\hline & 6 & 2.000 & 100 & 5,0 & +1 & -1 & +1 \\
\hline & 7 & 1.000 & 500 & 5,0 & -1 & +1 & +1 \\
\hline & 8 & 2.000 & 500 & 5,0 & +1 & +1 & +1 \\
\hline & 9 & 1.500 & 300 & 4,0 & 0 & 0 & 0 \\
\hline & 10 & 1.500 & 300 & 4,0 & 0 & 0 & 0 \\
\hline \multirow{10}{*}{2} & 11 & 800 & 400 & 3,0 & -1 & -1 & -1 \\
\hline & 12 & 1.200 & 400 & 3,0 & +1 & -1 & -1 \\
\hline & 13 & 800 & 600 & 3,0 & -1 & +1 & -1 \\
\hline & 14 & 1.200 & 600 & 3,0 & +1 & +1 & -1 \\
\hline & 15 & 800 & 400 & 4,0 & -1 & -1 & +1 \\
\hline & 16 & 1.200 & 400 & 4,0 & +1 & -1 & +1 \\
\hline & 17 & 800 & 600 & 4,0 & -1 & +1 & +1 \\
\hline & 18 & 1.200 & 600 & 4,0 & +1 & +1 & +1 \\
\hline & 19 & 1.000 & 500 & 3,5 & 0 & 0 & 0 \\
\hline & 20 & 1.000 & 500 & 3,5 & 0 & 0 & 0 \\
\hline \multirow{10}{*}{3} & 21 & 600 & 500 & 4,0 & -1 & -1 & - \\
\hline & 22 & 800 & 500 & 4,0 & -1 & +1 & - \\
\hline & 23 & 600 & 700 & 4,0 & +1 & -1 & - \\
\hline & 24 & 800 & 700 & 4,0 & +1 & +1 & - \\
\hline & 25 & 560 & 600 & 4,0 & $-1,4$ & 0 & - \\
\hline & 26 & 840 & 600 & 4,0 & $+1,4$ & 0 & - \\
\hline & 27 & 700 & 460 & 4,0 & 0 & $-1,4$ & - \\
\hline & 28 & 700 & 740 & 4,0 & 0 & $+1,4$ & - \\
\hline & 29 & 700 & 600 & 4,0 & 0 & 0 & - \\
\hline & 30 & 700 & 600 & 4,0 & 0 & 0 & - \\
\hline
\end{tabular}


Da mesma forma, para a técnica de coagulação-floculação-sedimentação, no delineamento experimental, apresentado na Tabela 4 , as variáveis independentes estudadas foram apenas a concentração de coagulante e o pH de coagulação.

\section{RESULTADOS E DISCUSSÃO}

\section{Processo Fenton}

As condições experimentais analisadas no planejamento inicial foram baseadas em ensaios preliminares, fornecendo a base para o desenvolvimento do segundo e terceiro planejamentos. A porcentagem de remoção de DQQ nesses dois planejamentos iniciais variou de $11 \%$ a $69 \%$. Essas variações são descritas a partir dos modelos matemáticos apresentados nas Equações 4 e 5, que correspondem ao primeiro e segundo planejamentos, respectivamente.

$$
\begin{gathered}
Y_{1}=32,47-1,96\left(X_{1}\right)+15,77\left(X_{2}\right)-4,18\left(X_{3}\right)- \\
1,09\left(X_{1} X_{2}\right)+2,49\left(X_{1} X_{3}\right)-0,32\left(X_{2} X_{3}\right)
\end{gathered}
$$

(Equação 4)

$$
\begin{gathered}
Y_{2}=58,43-1,93\left(X_{1}\right)+6,97\left(X_{2}\right)-1,52\left(X_{3}\right)+ \\
1,35\left(X_{1} X_{2}\right)-2,70\left(X_{1} X_{3}\right)+2,19\left(X_{2} X_{3}\right)
\end{gathered}
$$

(Equação 5)

Em que:

Y: porcentagem de remoção da DQO.

$\mathrm{X}_{1}$ : concentração de $\mathrm{H}_{2} \mathrm{O}_{2}$.

$\mathrm{X}_{2}$ : concentração de $\mathrm{Fe}^{2+}$.

\begin{tabular}{|c|c|c|c|c|c|}
\hline \multirow[b]{2}{*}{ Planejamento } & \multirow[b]{2}{*}{ Ensaio } & \multicolumn{2}{|c|}{ Variável } & \multicolumn{2}{|c|}{ Variável codificada } \\
\hline & & $\begin{array}{l}\text { Dosagem de } \\
\text { coagulante } \\
\left(\mathrm{mg} \mathrm{Fe}^{3+} \mathrm{L}^{-1}\right)\end{array}$ & pH & $\begin{array}{l}\text { Dosagem de } \\
\text { coagulante } \\
\left(\mathrm{mg} \mathrm{Fe}^{3+} \mathrm{L}^{-1}\right)\end{array}$ & pH \\
\hline \multirow{12}{*}{1} & 1 & 300 & 4,0 & -1 & -1 \\
\hline & 2 & 300 & 6,0 & -1 & +1 \\
\hline & 3 & 500 & 4,0 & +1 & -1 \\
\hline & 4 & 500 & 6,0 & +1 & +1 \\
\hline & 5 & 260 & 5,0 & $-1,4$ & 0 \\
\hline & 6 & 540 & 5,0 & $+1,4$ & 0 \\
\hline & 7 & 400 & 3,6 & 0 & $-1,4$ \\
\hline & 8 & 400 & 6,4 & 0 & $+1,4$ \\
\hline & 9 & 400 & 5,0 & 0 & 0 \\
\hline & 10 & 400 & 5,0 & 0 & 0 \\
\hline & 11 & 400 & 5,0 & 0 & 0 \\
\hline & 12 & 400 & 5,0 & 0 & 0 \\
\hline \multirow{6}{*}{2} & 13 & 150 & 4,0 & -1 & -1 \\
\hline & 14 & 150 & 6,0 & -1 & +1 \\
\hline & 15 & 350 & 4,0 & +1 & -1 \\
\hline & 16 & 350 & 6,0 & +1 & +1 \\
\hline & 17 & 250 & 5,0 & 0 & 0 \\
\hline & 18 & 250 & 5,0 & 0 & 0 \\
\hline \multirow{4}{*}{ Ensaios adicionais } & 19 & 150 & 5,0 & & \multirow{4}{*}{-} \\
\hline & 20 & 250 & 4,0 & & \\
\hline & 21 & 250 & 6,0 & & \\
\hline & 22 & 350 & 5,0 & & \\
\hline
\end{tabular}

$X_{3}: \mathrm{pH}$.

Tabela 4 - Variáveis e níveis definidos para o estudo da coagulação-floculação-sedimentação como pós-tratamento de lixiviado de aterro sanitário utilizando a técnica de planejamento fatorial de experimentos. 
Tabela 5 - Valores residuais de DQ̣O e porcentagem de remoção para a aplicação de processo Fenton após o tratamento biológico - Terceiro planejamento.

\begin{tabular}{|c|c|c|c|c|}
\hline \multirow[b]{2}{*}{ Ensaio } & \multicolumn{2}{|c|}{ Variável } & \multicolumn{2}{|c|}{ Variável-resposta } \\
\hline & $\begin{array}{c}{\left[\mathrm{H}_{2} \mathrm{O}_{2}\right]} \\
\left(\mathrm{mg} \mathrm{L}^{-1}\right)\end{array}$ & $\begin{array}{c}{\left[\mathrm{Fe}^{2+}\right]} \\
\left(\mathrm{mg} \mathrm{L}^{-1}\right)\end{array}$ & $\begin{array}{l}\text { DQO residual } \\
\left(\mathrm{mg} \mathrm{O}_{2} \mathrm{~L}^{-1}\right)\end{array}$ & \% remoção \\
\hline 21 & 600 & 500 & 660 & 59 \\
\hline 22 & 800 & 500 & 629 & 61 \\
\hline 23 & 600 & 700 & 492 & 70 \\
\hline 24 & 800 & 700 & 473 & 71 \\
\hline 25 & 560 & 600 & 569 & 65 \\
\hline 26 & 840 & 600 & 551 & 66 \\
\hline 27 & 700 & 460 & 791 & 51 \\
\hline 28 & 700 & 740 & 523 & 68 \\
\hline 29 & 700 & 600 & 525 & 68 \\
\hline 30 & 700 & 600 & 529 & 67 \\
\hline
\end{tabular}

Nota: * DQQO inicial média = $1.624 \mathrm{mg} \mathrm{O2} \mathrm{L-1.}$

No primeiro planejamento, observou-se que apenas a concentração de $\mathrm{Fe}^{2+}$ teve efeito significativo sobre a remoção de DQQO. Já no segundo planejamento, nas condições experimentais testadas, as três variáveis envolvidas afetaram de maneira significativa a variável-resposta, com nível de significância de 5\% (p-valor<0,05). Os modelos matemáticos que descrevem o processo, apresentados nas Equações 4 e 5, são capazes de explicar cerca de $99 \%\left(R^{2}=0,99\right)$ das variações na remoção de DQQO.

De acordo com as equações, a dosagem de $\mathrm{Fe}^{2+}$ $\left(\mathrm{X}_{2}\right)$ é a variável independente de maior efeito sobre a variável-resposta, atuando positivamente sobre a remoção de DQQO, ou seja, para maiores dosagens, maior a porcentagem de remoção. $\mathrm{Da}$ mesma forma, dentro das condições experimentais estudadas, a concentração de peróxido de hidrogênio $\left(-1,93 X_{1}\right)$ exerce efeito negativo (menores dosagens, maior remoção de DQQO) e de maneira menos significativa em relação à concentração de $\mathrm{Fe}^{2+}$.

Com base nessas considerações, foi realizado o terceiro planejamento, no qual a dosagem de $\mathrm{Fe}^{2+}$ foi elevada, a concentração de peróxido de hidrogênio foi reduzida e o $\mathrm{pH}$ foi fixado em 4,0, tendo em vista seu efeito pouco significativo sobre o processo nas condições estudadas.

Os resultados de remoção de DQQO obtidos para o terceiro planejamento fatorial são apresentados na Tabela 5.

Conforme mostra a Tabela 6, a concentração de $\mathrm{Fe}^{2+}$ foi a única variável com efeito significativo sobre a variável-resposta, com $5 \%$ de significância.

Tabela 6 - Resultados da análise de variância para a variável-resposta remoção de DQQO pelo processo Fenton - terceiro planejamento.

\begin{tabular}{|c|c|c|}
\hline Fatore & Efeito & p-valor \\
\hline$\left[\mathrm{H}_{2} \mathrm{O}_{2}\right]\left(\mathrm{mg} \mathrm{L}^{-1}\right)(\mathrm{L})^{*}$ & 1,10355 & 0,492316 \\
\hline$\left[\mathrm{H}_{2} \mathrm{O}_{2}\right]\left(\mathrm{mg} \mathrm{L}^{-1}\right)(\mathrm{Q})^{* * *}$ & $-0,62500$ & 0,762740 \\
\hline$\left[\mathrm{Fe}^{2+}\right]\left(\mathrm{mg} \mathrm{L}^{-1}\right)(\mathrm{L})^{* *}$ & 11,26041 & 0,001528 \\
\hline$\left[\mathrm{Fe}^{2+}\right]\left(\mathrm{mg} \mathrm{L}^{-1}\right)(\mathrm{Q})^{* * *}$ & $-6,62500$ & 0,026637 \\
\hline$\left[\mathrm{H}_{2} \mathrm{O}_{2}\right] \times\left[\mathrm{Fe}^{2+}\right]^{*}$ & $-0,5000$ & 0,820786 \\
\hline
\end{tabular}

Notas: *(L) Efeitos lineares. **(Q) Efeitos quadráticos.

O modelo, representado pela Equação 6, é capaz de explicar $99 \%$ das variações da remoção de 
DQO pelo processo Fenton, no qual se observa o efeito sobressalente da concentração de $\mathrm{Fe}^{2+}$ sobre a variável-resposta.

$$
\begin{gathered}
Y_{3}=67,50+0,55\left(X_{1}\right)-0,31\left(X_{1}\right)^{2}+5,63\left(X_{2}\right)- \\
3,31\left(X_{2}\right)^{2}-0,25\left(X_{1} X_{2}\right)
\end{gathered}
$$

(Equação 6)

Em que:

Y: porcentagem de remoção da DQQ.

$\mathrm{X}_{1}$ : concentração de $\mathrm{H}_{2} \mathrm{O}_{2}$.

$\mathrm{X}_{2}$ : concentração de $\mathrm{Fe}^{2+}$.

Ao analisar os resultados estatísticos do planejamento fatorial simultaneamente à superfície de resposta, é evidenciado, de acordo com a Figura 1, que a concentração de peróxido de hidrogênio já não influencia de maneira significativa a remoção de DQO, o que pode ser verificado ao comparar os experimentos 23 e 24 da Tabela 5. Além disso, para essa condição experimental, a remoção está sendo muito mais influenciada pela dosagem de $\mathrm{Fe}^{2+}$ e, portanto, pelo fenômeno de coagulação. Comparando o ensaio 17 com o 24 (Tabela 5), verificou-se que, para a mesma concentração de peróxido de hidrogênio de $800 \mathrm{mg} \mathrm{L}^{-1}$ e dosagem de $\mathrm{Fe}^{2+}$ de $600 \mathrm{mg} \mathrm{L}^{-1}$ (Ensaio 17) para $700 \mathrm{mg} \mathrm{L}^{-1}$

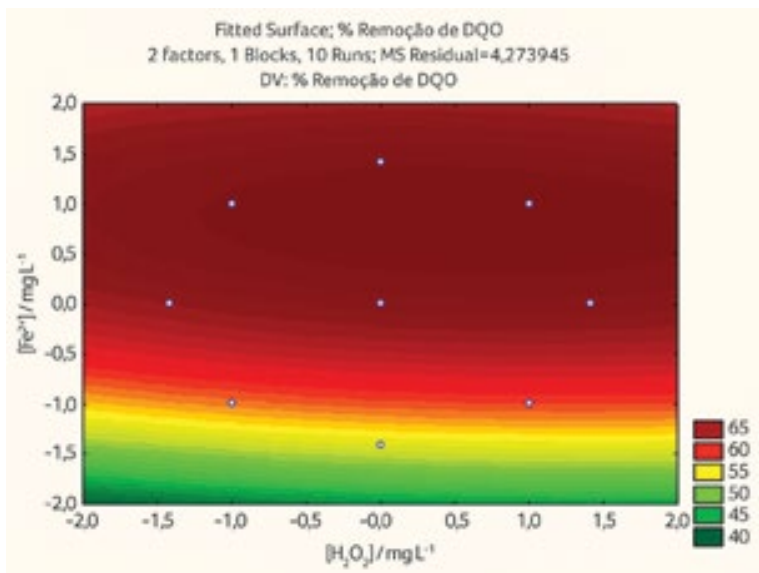

Figura 1 - Superfície de contorno para a variável-resposta porcentagem de remoção de DQQO para as variáveis [H2O2] e [Fe2+] - terceiro planejamento.
(Ensaio 24), a remoção de DQO passou apenas de $69 \%$ para $71 \%$. Entretanto, o experimento $23 \mathrm{da}$ Tabela 5 mostra que, trabalhando com a mesma dosagem de $\mathrm{Fe}^{2+}$ de $700 \mathrm{mg} \mathrm{L}^{-1}$ e reduzindo o teor de peróxido de hidrogênio para $600 \mathrm{mg} \mathrm{L}^{-1}$, já se atinge remoção de DOQ de $70 \%$.

A remoção máxima de $\mathrm{DQ} O$ foi de $71 \%$ quando se utilizaram $800 \mathrm{mg} \mathrm{L}^{-1}$ de peróxido de hidrogênio e $700 \mathrm{mg} \mathrm{L}^{-1}$ de $\mathrm{Fe}^{2+}$, ou seja, com a razão $\left[\mathrm{H}_{2} \mathrm{O}_{2}\right] /$ $\left[\mathrm{Fe}^{2+}\right]=1,14$. Tal valor não difere significativamente do resultado mencionado anteriormente, obtido quando se utilizaram $600 \mathrm{mg} \mathrm{L}^{-1}$ de peróxido de hidrogênio e $700 \mathrm{mg} \mathrm{L}^{-1}$ de $\mathrm{Fe}^{2+}$, condição na qual a concentração superior do $\mathrm{Fe}^{2+}$ em relação ao peróxido de hidrogênio elimina sua atuação de apenas catalisar a reação, reforçando sua atuação como coagulante. Pode-se concluir que, de modo geral, os resultados obtidos para a remoção de DOQO por processo Fenton neste trabalho são atribuídos predominantemente ao processo de coagulação.

Resultados similares foram obtidos por $\mathrm{Li}$ et al. (2010), que, a partir de uma razão $\left[\mathrm{H}_{2} \mathrm{O}_{2}\right] /\left[\mathrm{Fe}^{2+}\right]$ também relativamente baixa $(2,38)$, removeram até $72,8 \%$ da DQO de lixiviado previamente tratado em sistema de lodos ativados em batelada sequencial. A análise estatística aliada à metodologia de superfície de resposta indicou que a concentração de peróxido de hidrogênio teve o maior efeito sobre a variável-resposta remoção de DQQO, ao passo que, ao admitir a remoção de cor como variável-resposta, o $\mathrm{Fe}^{2+}$ foi o principal atuante.

Kang et al. (2000) relataram que o pH ótimo para remoção de DQQO por oxidação pelo processo Fenton se encontra abaixo de 4,0. Sob as condições ótimas de ensaio $\left(\mathrm{H}_{2} \mathrm{O}_{2}=1.650 \mathrm{mg} \mathrm{L}^{-1}\right)$, foi observado que cerca de $45 \%$ da DQO foi removida por oxidação, ao passo que aproximadamente $20 \%$ foram removidos por coagulação. Segundo esses autores, a elevação do $\mathrm{pH}$ levou à maior atuação do processo de coagulação em relação ao de oxidação na remoção de DQ̣O. 
Tabela 7 - Valores residuais de DQ̨O e porcentagem de remoção para a aplicação da coagulação-floculação-sedimentação após o tratamento biológico - segundo planejamento.

\begin{tabular}{|c|c|c|c|c|}
\hline \multirow[b]{2}{*}{ Ensaio } & \multicolumn{2}{|c|}{ Variável } & \multicolumn{2}{|c|}{ Variável-resposta } \\
\hline & $\begin{array}{l}\text { Dosagem de coagulante } \\
\qquad\left(\mathrm{mg} \mathrm{Fe}^{++} \mathrm{L}^{-1}\right)\end{array}$ & pH & $\begin{array}{l}\text { DQO residual } \\
\left.\text { ( } \mathrm{mg} \mathrm{O}_{2} \mathrm{~L}^{-1}\right)\end{array}$ & \% remoção \\
\hline 13 & 150 & 4,0 & 558 & 72 \\
\hline 14 & 150 & 6,0 & 1.224 & 39 \\
\hline 15 & 350 & 4,0 & 440 & 78 \\
\hline 16 & 350 & 6,0 & 1.014 & 52 \\
\hline 17 & 250 & 5,0 & 511 & 75 \\
\hline 18 & 250 & 5,0 & 463 & 77 \\
\hline
\end{tabular}

Nota: "DQQO inicial média $=2.014 \mathrm{mg} \mathrm{O}_{2} \mathrm{~L}^{-1}$.

\section{Coagulação-floculação-sedimentação}

O primeiro planejamento consistiu num planejamento DCCR $2^{3}$, tendo como variáveis independentes a dosagem de $\mathrm{Fe}^{3+}$ e o $\mathrm{pH}$. $\mathrm{O}$ modelo matemático que explica as alterações sofridas pela variável-resposta no primeiro planejamento realizado é apresentado na Equação 7.

$$
\begin{gathered}
Y_{1}=68,50+1,46\left(X_{1}\right)+0,81\left(X_{1}\right)^{2}- \\
8,38\left(X_{2}\right)-3,44\left(X_{2}\right)^{2}
\end{gathered}
$$

(Equação 7)

Em que:

Y: porcentagem de remoção da DQQO.

$\mathrm{X}_{1}$ : dosagem de $\mathrm{Fe}^{3+}$.

$\mathrm{X}_{2}: \mathrm{pH}$.

De acordo com a equação, a variável $\mathrm{pH}$ tem sobre a variável-resposta um efeito cerca de seis vezes maior que a dosagem de $\mathrm{Fe}^{3+}$. Isso sugere que o ajuste de $\mathrm{pH}$ pode ser responsável por grande parte da remoção de DQO por meio da precipitação de componentes insolúveis do lixiviado nos valores de $\mathrm{pH}$ testados.

Com o intuito de avaliar o efeito de dosagens inferiores de $\mathrm{Fe}^{3+}$ sobre o processo, o segundo planejamento foi realizado reduzindo a faixa de valores dessa variável de 150 para $350 \mathrm{mg} \mathrm{L}^{-1}$, mantendo a faixa de $\mathrm{pH}$ entre 4,0 e 6,0. Os resultados obtidos são apresentados na Tabela 7.
Conforme a Tabela 7, os menores valores de remoção de DQQ (39\% e 52\%) foram obtidos com pH superior $(6,0)$, ao passo que, com $\mathrm{pH} 4,0$, se obteve até $78 \%$ de remoção, resultando numa $D Q Q$ residual de $440 \mathrm{mg} \mathrm{O}_{2} \mathrm{~L}^{-1}$. O efeito do $\mathrm{pH}$ no sentido negativo pode ser observado pela análise estatística dos resultados (Tabela 8), na qual se constatou que esta é a variável de maior influência sobre a remoção de DQQO.

Tabela 8 - Resultados da análise de variância para a variável-resposta remoção de DQQO por coagulaçãofloculação-sedimentação - segundo planejamento.

\begin{tabular}{|c|c|c|}
\hline Fator & Efeito & p-valor \\
\hline$\left[\mathrm{Fe}^{3+}\right]$ & 9,500 & 0,538094 \\
\hline $\mathrm{pH}$ & $-29,50$ & 0,149471 \\
\hline$\left[\mathrm{Fe}^{3+}\right] \times \mathrm{pH}$ & 3,50 & 0,811566 \\
\hline
\end{tabular}

O modelo estatístico apresentado na Equação 8 é capaz de explicar $99 \%$ das variações da remoção de DQQO pelo processo de coagulação-floculação-sedimentação, no qual se observa o efeito sobressalente do $\mathrm{pH}$ sobre a variável-resposta.

$$
\mathrm{Y}_{2}=65,50+4,75\left(\mathrm{X}_{1}\right)-14,75\left(\mathrm{X}_{2}\right)+1,75\left(\mathrm{X}_{1} \mathrm{X}_{2}\right)
$$

(Equação 8)

\section{Comparação entre os dois processos nas condições estudadas}

Comparando os resultados mais favoráveis para os dois processos nas condições estudadas, observa-se que o $\mathrm{pH}$ ótimo foi de 4,0. Nessa situação, o processo Fenton reduziu a DQQO de 1.624 mg 


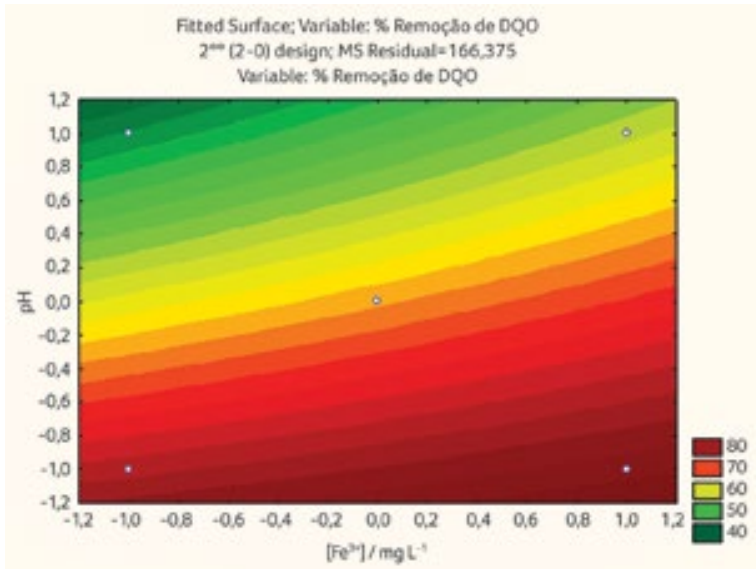

Figura 2 - Superfície de contorno para a variável-resposta porcentagem de remoção de DQQO para as variáveis $[\mathrm{pH}]$ e $[\mathrm{Fe} 3+]$ - segundo planejamento.

$\mathrm{O}_{2} \mathrm{~L}^{-1}$ para $473 \mathrm{mgO}_{2} \mathrm{~L}^{-1}$, apresentando eficiência de $71 \%$. A cor verdadeira foi reduzida de $3.590 \mathrm{uH}$ para 320 uH (91\% de remoção).

No caso do tratamento por coagulação-floculação-sedimentação, a DQQO foi reduzida de 2.014 $\mathrm{mg} \mathrm{O}_{2} \mathrm{~L}^{-1}$ para $440 \mathrm{mgO}_{2} \mathrm{~L}^{-1}$ (78\% de remoção). Já a cor verdadeira passou de $3.500 \mathrm{uH}$ para $140 \mathrm{uH}$, ou seja, $96 \%$ de remoção.

No âmbito deste trabalho, as relações $\left[\mathrm{H}_{2} \mathrm{O}_{2}\right] /\left[\mathrm{Fe}^{2+}\right]$ foram definidas com base no planejamento fatorial apresentado. Outros trabalhos científicos (LI et al, 2010; DENG et al, 2006; LOPEZ et al., 2004) apresentam dados com relação $\left[\mathrm{H}_{2} \mathrm{O}_{2}\right] /\left[\mathrm{Fe}^{2+}\right]$ superior a estas, levando a taxas maiores de oxidação devido à ação do peróxido de hidrogênio.

A formação de lodo não foi estudada em detalhes, porém se observou, ao comparar os melhores resultados obtidos para os dois processos, que o volume de lodo gerado pelo processo Fenton foi aproximadamente metade do volume gerado pelo tratamento físico-químico convencional. Tal volume de lodo foi obtido, sem variações posteriores, em 30 minutos para o processo Fenton e em 2 horas para a coagulação-floculação-sedimentação, concluindo-se, portanto, que a velocidade de sedimentação do lodo formado pelo primeiro processo foi maior que a do segundo.
Como o lixiviado já havia passado pelos tratamentos de arraste e lodos ativados em batelada sequencial, o nitrogênio já havia sido removido.

Os dois processos utilizados como pós-tratamento (Fenton e coagulação-floculação-sedimentação) apresentaram boa eficiência. Do ponto de vista prático, o tratamento por coagulação-floculação-sedimentação dispõe no mercado de equipamentos que permitem um projeto mais seguro considerando a engenharia, enquanto o processo Fenton carece de um histórico de desenvolvimento em escala real.

Por outro lado, a adição de elevadas dosagens de cloreto férrico agrava ainda mais a concentração de cloretos presentes no lixiviado. Kawahigashi (2010), em condições semelhantes, realizou ensaios de ecotoxicidade com os organismos-teste Ceriodaphnia dubia, Daphnia magna, Pseudokirchneriella subcapitata e Artemia salina, sendo observado o aumento da toxicidade no efluente após tratamento por coagulação-floculação-sedimentação. A concentração efetiva do efluente inicial foi reduzida de valores entre 20,6 e 30,1 para valores que variaram de 0,2 a 9,6 após o tratamento. Tal alteração pode ser relacionada aos residuais de metais, como ferro, além do aumento do residual de cloretos no lixiviado de $2.744 \mathrm{mg} \mathrm{L}^{-1}$ para valores da ordem de $4.000 \mathrm{mg} \mathrm{L}^{-1}$ após o tratamento, em virtude da utilização de cloreto férrico como coagulante.

\section{CONCLUSÕES}

Tendo em vista o tipo de lixiviado utilizado neste trabalho e o tratamento prévio a que foi submetido (stripping e lodo ativado em batelada com nitrificação-desnitrificação) e considerando que as relações $\left[\mathrm{H}_{2} \mathrm{O}_{2}\right] /\left[\mathrm{Fe}^{2+}\right]$ foram definidas com base no planejamento fatorial e respectivas superfícies de resposta, pode-se concluir que:

- o pós-tratamento do lixiviado de aterro sanitário por coagulação-floculação-sedimentação permitiu a remoção de até $96 \%$ da cor ver- 
dadeira e $78 \%$ da DQO quando se utilizaram $350 \mathrm{mg} \mathrm{L}^{-1}$ de $\mathrm{Fe}^{3+}$ em pH 4,0;

- o processo Fenton removeu até $91 \%$ de cor verdadeira e $71 \%$ de DQO ao utilizar $800 \mathrm{mg}$ $\mathrm{L}^{-1}$ de peróxido de hidrogênio e $600 \mathrm{mg} \mathrm{L}^{-1}$ de $\mathrm{Fe}^{2+}$, em pH 4,0;

- no âmbito deste trabalho, a concentração de $\mathrm{Fe}^{2+}$ foi a variável de maior efeito sobre a remoção de DQQO quando se utilizou o processo Fenton, ao passo que o $\mathrm{pH}$ exerceu maior influência no caso da coagulação-floculação-sedimentação;

- embora não tenha sido objeto deste trabalho, observou-se que o lodo gerado pelo processo Fenton apresentou maior velocidade de sedimentação e menor volume em relação à coagulação-floculação-sedimentação.

\section{AGRADECIMENTOS}

Os autores agradecem à Coordenação de Aperfeiçoamento de Pessoal de Nível Superior (Capes) pela concessão de auxílio - bolsa de estudo de mestrado -, ao Programa de Mestrado em Engenharia de Edificações e Saneamento da UEL e à Financiadora de Estudos e Projetos (Finep)/ Ministério da Ciência e Tecnologia (MCT) (Edital Saneamento Ambiental e Habitação - 7/2009).

\section{REFERÊNCIAS}

ALVIM, C. A. N. Influências do pré-tratamento por stripping na desnitrificação convencional e pela via curta, em RBS aplicado ao tratamento de lixiviado de aterro sanitário. Dissertação (Mestrado). Universidade Estadual de Londrina, 2008).

APHA, AWWA, WEF (2005). Standards Methods for the Examination of Water e Wastewater, $21^{\circ} \mathrm{ed}$. Washington. D.C.

DENG, Y.; ENGLEHARDT, J. D. Treatment of landfill leachate by Fenton process. Water Research, v. 40, p. 3683-3694, 2006.

DI BERNARDO, L.; DANTAS DI BERNARDO, A. Métodos e Técnicas de Tratamento de Água. Volume 1, São Carlos - 2005. Métodos Técnicas Água - 1 - $2^{\mathrm{a}}$ edição

FELICI, E. M.; KURODA, E. K.; YAMASHITA, F.; SILVA, S. M. C. P. Remoção de carga orgânica recalcitrante de lixiviado de resíduos sólidos urbanos pré-tratado biologicamente por coagulação quími- ca-floculação-sedimentação. Engenharia Sanitária e Ambiental, v. 18, p. 177-184, 2013.

HOSSAKA, A. L.; FERNANDES, F.; SILVA, S. M. C. P.; LOPES, D. D. Evaluation of nitrite acumulation in biological treatment systems, aiming at shortcut denitrification. Acta Scientiarum: Technology, v. 34, p. 261-267, 2012.

KANG, Y. W.; CHO, M.; HWANG, K. Correction of hydrogen peroxide interference on standard chemical oxygen demand test. Water Research, v. 33, p. 1247-1251, 1999.

KANG, Y. W.; HWANG, K. Effects of reaction conditions on the oxidation efficiency in the Fenton process. Water Research, v. 34, p. 2786-2790, 2000.

KAWAHIGASHI, F. Aplicabilidade do pós-tratamento de lixiviado de aterro sanitário por adsorção em carvão ativado granular e avaliação ecotoxicológica. Dissertação (Mestrado). Dissertação apresentada ao Programa de Mestrado em Engenharia de Edificações e Saneamento.Universidade Estadual de Londrina, 2012.

KJELDSEN, P.; BARLAZ, M. A.; ROOKER, A. P.; BAUN, A.; LEDIN, A.; CHRISTENSEN, T. H. Present and long-term composition of MSW landfill leachate: areview. Critical Reviews in Environmental Science and Technology, v. 32, n. 4,p. 297-336, 2002.

LANGE, L. C.; ALVES, J. F.; AMARAL, M. C. S.; MELO JÚNIOR, W. R. Tratamentode lixiviado de aterro sanitário por processo oxidativo avançado empregando reagente de Fenton. Engenharia Sanitária e Ambiental, v. 11, p. 175-183, 2006.

LI, H.; ZHOU, S.; SUN, Y.; LV, J. Application of response surface methodology to the advanced treatment of biologically stabilized landfill leachate using Fenton's reagent. Waste Management, 30, p. 2122-2129, 2010.

LOPEZ, A.; PAGANO, M.; VOLPE, A.; DI PINTO, A. Fenton's pre-treatment of mature landfill leachate. Chemosphere, v. 54, p. 10001005, 2004.

MALER, C. L. Aplicação do Processo Fenton a diferentes etapas do tratamento de lixiviado de aterro sanitário. Dissertação (Mestrado). Dissertação apresentada ao Programa de Mestrado em Engenharia de Edificações e Saneamento.Universidade Estadual de Londrina, 2013.

MORAVIA, W. G.; LANGE, L. C.; AMARAL, M. C. S. Avaliação de processo oxidativo avançado pelo reagente de Fenton em condições otimizadas no tratamento de lixiviado de aterro sanitário com ênfase em parâmetros coletivos e caracterização do lodo gerado. Química Nova, v. 34, p. 1370-1377, 2011.

PERALTA-ZAMORA, P.; ESPOSITO, E.; REYES, H. D.; DURAN, N. Remediação de efluentes derivados da indústria de papel e celulose. Tratamento biológico e fotocatalítico. Química Nova, v. 20, n. 2, p. 186-190, 1997.

RENOU, S., GIVAUDAN, J. G., POULAIN, S., DIRASSOUYAN, F., MOULIN, P. Landfill leachate treatment: Review and opportunity. Journal of Hazardous Materials, v. 150, p. 468-493, 2008. 